\title{
Impact social des délocalisations: mythe ou réalité?
}

\author{
Elwardi Dhaoui \\ (Unité de recherche Monnaie, Développement et Infrastructures/ Faculté des Sciences Économiques et de \\ Gestion de Sfax, Tunisie)
}

\begin{abstract}
Résumé: Cet article tente d'apporter une validation empirique de la mondialisation financière. Dans un premier lieu, on va donner une idée sur les réorganisations des fonctions de production des entreprises mondiales et on va insister sur l'ampleur du phénomène. De même, on va converser si le fait de l'importance de la taille traduit la loi du plus fort. En second lieu, on va discuter les conséquences pour l'emploi de la multinationalisation et de la délocalisation dans les pays du Nord et dans les pays du Sud. Alors, comment définir les délocalisations? Quelles sont les principaux motifs de délocalisation? Quel est l'ampleur de ce phénomène? C'est-à-dire y a-t-il une accélération des délocalisations au cours de la période récente? Comment évaluer les bénéfices tirés des délocalisations, notamment en termes d'emploi? Quelles sont les catégories d'emplois, les secteurs et les pays les plus touchés? Quelles sont les réponses des politiques et quelles mesures peut-on préconiser pour réduire les cô̂ts, faciliter l'ajustement et rétablir la confiance?
\end{abstract}

Mots-clés - investissement direct à l'étranger, firmes transnationales, emploi, pays développés, pays émergents.

\section{Introduction}

Un fait marquant est que les années 80 ont vu se constituer un véritable marché mondial de capitaux. Jamais dans l'histoire, l'épargne accumulée dans un territoire quelconque n'avait en autant de facilité à aller s'investir dans un autre. Cette mondialisation financière est véhiculée par les firmes multinationales qui adoptent de plus en plus des stratégies globales dans l'organisation de leurs produits et de leurs échanges.

Par ailleurs, l'accroissement rapide du poids des entreprises multinationales dans l'économie mondiale soulève des préoccupations nouvelles pour les économistes, mais aussi pour les responsables politiques et la société civile dans son ensemble.

L'étude du rôle des multinationales dans le commerce international permet de mieux comprendre leur impact sur le développement et surtout l'emploi dans des pays d'origine et d'accueil. Les délocalisations et implantations des firmes à l'étranger constituent un mouvement étroitement lié à la mondialisation de l'économie marquée par une globalisation du processus de production des firmes multinationales, une déréglementation des marchés financiers (offrant une plus grande souplesse dans les différentes opérations menées par les entreprises). La conjonction de ces éléments explique, pour une large part, les opérations de fusions-acquisitions qui se développent à l'échelle mondiale et qui n'épargnent aucun secteur d'activité. S'y ajoute une intégration croissante des économies nationales au sein d'espaces régionaux tels que l'Alena, l'Union européenne ou encore la zone Asie-Pacifique. Malgré cette globalisation des économies et des échanges, des disparités nationales et régionales, surtout en termes d'emplois apparaissent.

\section{II.1. Définition}

\section{Les IDE}

L'IDE est une opération par laquelle un investisseur basé dans un pays (pays d'origine) acquiert un actif dans un autre pays (pays d'accueil) avec l'intention de le gérer.

Notons que cette notion est différente de celle de l'investissement de portefeuille (prise de participation minoritaire dans le capital d'une entreprise. La différence entre ces deux notions est basée sur un critère de taux de participation ${ }^{1}$.

\section{II.2. Les modalités d'IDE}

Les méthodes de l'implantation à l'étranger pour une entreprise sont multiples.

Principalement, on peut citer:

- La cession de licence : c'est un contrat par lequel une entreprise d'un pays concède à une entreprise située dans un autre pays le droit d'utiliser ou d'exploiter un processus de fabrication ;

\footnotetext{
${ }^{1}$ Il s'agit d'un critère propre au FMI, la valeur seuil est de $10 \%$ du capital.
} 
- La création de filiales dans le pays d'accueil ;

- Joint-venture : alliance d'entreprises sous la forme d'une filiale commune ;

- L'acquisition totale ou partielle d'entreprises déjà établies : on parle des opérations de fusion et d'acquisition ;

- La sous-traitance : consiste à se concentrer sur une activité principale et à faire réaliser par des intervenants extérieurs des ouvrages qui font partie des activités secondaires.

\section{II.3. La stratégie de délocalisation}

\section{II.3.1. Importance de la délocalisation}

Le phénomène de multinationalisation est le résultat d'interférence entre l'investissement direct, la soustraitance avec des firmes non affiliées et le commerce international. En général, tant l'investissement direct que le recours à la sous-traitance à l'étranger impliquent des flux commerciaux, notamment des importations vers le pays d'origine mais également des exportations.

L'importance de la délocalisation résulte des avantages compétitifs du pays qui prennent non seulement les coûts comparés des facteurs, mais également les avantages comparés de la taille et des dynamiques de demande nationale. En effet, une extension de la production permet de bénéficier d'économies d'échelle sur un marché étranger. Le transfert par les firmes transnationales des activités de production d'un pays vers un autre constitue désormais une question importante en matière de relations économiques entre les pays.

\section{II.3.2. Les déterminants de la délocalisation}

Les déterminants de la délocalisation sont multiples, parmi lesquels le coût du travail, le coût du transport, l'infrastructure, la main d'œuvre qualifiée, etc.

Les raisons qui poussent les entreprises à délocaliser peuvent être différentes selon le secteur et la forme que prend la délocalisation.

Fig. 1 : Les motivations pour se délocaliser (en \% des réponses)

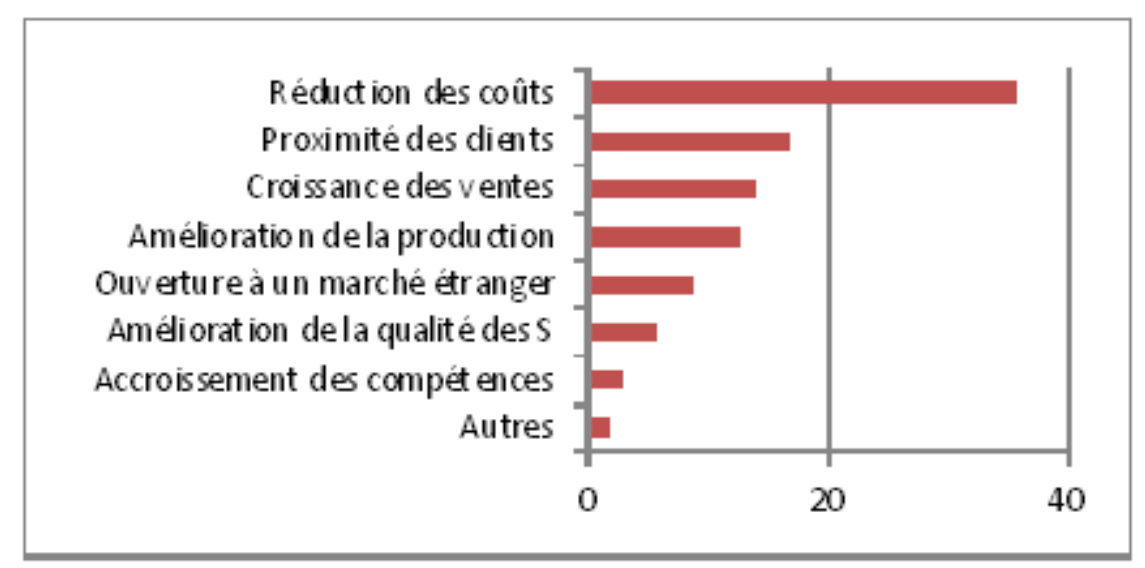

Source : Les délocalisations et l'emploi : tendances et impacts, OCDE 2007.

\section{II.3.2.1. Le coût du travail : le principal déterminant}

Le coût du travail est la variable non exclusive mais déterminante de la délocalisation.

En effet, toute la théorie économique de la spécialisation internationale et de la division du travail, repose au départ sur le coût des facteurs de production ${ }^{2}$.

L'analyse suit deux types : la spécialisation puis la délocalisation.

$>$ étape $1:$ la spécialisation

Un pays se spécialise dans les productions pour lesquelles il bénéficie d'un avantage comparatif en termes de coût. Les principes ont été étudiés depuis le XVIII ${ }^{\text {ème }}$ siècle, et même devenus plus sophistiqués, gardent toute leur pertinence aujourd'hui.

$>$ étape 2 : la délocalisation

L'analyse théorique de la délocalisation est entendue au sens strict, c'est-à-dire production dans un pays et réimportation, combine la théorie générale des coûts comparatifs avec la théorie des avantages compétitifs des firmes.

\footnotetext{
${ }^{2}$ J.L. Muchielli a beaucoup travaillé sur ce point.
} 
Selon Arthuis (1993), ces avantages compétitifs peuvent provenir de deux sources, celles qui réduisent les coûts de production (par innovation technologique) et celle qui permettent la différenciation des produits (marque, publicité).

Par ailleurs, les entreprises sont très tentées par la délocalisation car cette politique engendre plusieurs avantages notamment d'ordre économique. En effet, lorsqu'une entreprise délocalise, elle voit, et c'est l'enjeu majeur d'une délocalisation, les salaires des employés très souvent inférieurs à celui des pays d'origine. Les coûts de production deviennent moindres, ce qui se traduit par une meilleure compétitivité de l'entreprise.

Les enjeux majeurs d'une délocalisation sont donc au niveau financière, c'est pour cela que les entreprises choisissent les pays où les salaires sont très faibles.

Aujourd'hui, être compétitif sur le plan mondial c'est offrir un produit avec un coût compétitif par rapport aux autres; c'est dans cette esprit que plusieurs entreprises cherchent à se déplacer pour bénéficier des mains d'œuvre abondants et à faible coût. Donc, les différentiels des salaires entre diverses régions géographiques peuvent être importants.

\section{II.3.2.2. Les autres déterminants}

D'autres raisons peuvent inciter les entreprises à délocaliser ; parmi lesquels on peut citer :

La disponibilité des compétences et de mains d'œuvre qualifiées.

- La proximité des marchés : proximité des clients potentiels dans une région de monde ;

- L'existence d'une infrastructure développée ;

- Les cadres règlementaires (législation du travail, les avantages fiscaux, etc.).

\subsection{Un arbitrage simple entre les stratégies d'exportation et celle de délocalisation}

L'IDE est du ressort des FTN pour laquelle un choix stratégique s'impose : est-il préférable d'exporter des produits crées localement ou faut-il privilégier l'accès au marché par le biais des IDE ?

En effet, l'exportation permet d'économiser les coûts fixes d'implantation d'une filiale à l'étranger ; mais il faut payer les coûts variables du transport.

De sa part, l'IDE exige le paiement des coûts fixes d'implantation ; mais il économise les coûts variables.

On comprend donc que l'IDE sera privilégié si le marché est grand, protégé par des barrières douanières et si les coûts fixes d'installation sont faibles.

L'IDE est vu ici comme un substitut au commerce.

\section{Le Bilan des faits}

\section{III.1. L'intégration des marchés financiers}

Un élément de mesure de degré d'intégration des marchés financiers mondiaux consiste à examiner si une nation peu facilement être financée par les capitaux étrangers ${ }^{3}$.

Fig.2: L'intégration financière internationale

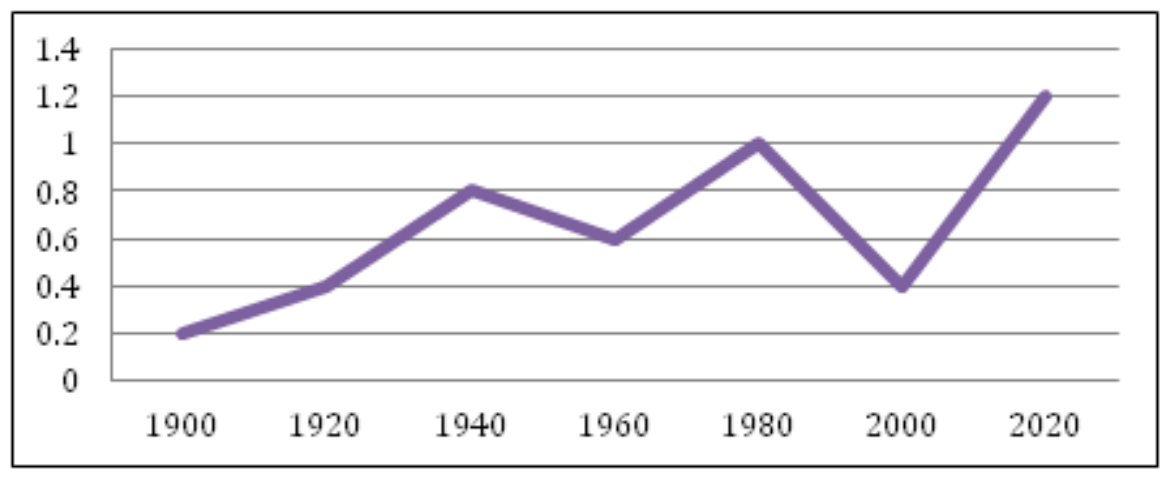

Source: Flandreau $C$. et Rivière C., La grande retransformation: intégration financière internationale et contrôle de capitaux, Économie Internationale, $n^{0}$ 78, 1999.

\footnotetext{
${ }^{3}$ Il faut pour cela se pencher sur la relation entre épargne nationale et investissement national. Dans un monde segmenté, le niveau de l'investissement national est contraint par le montant de l'épargne nationale, et la corrélation entre épargne nationale et investissement nationale est donc plus lâche. On peut donc déduire d'une mesure du degré de corrélation entre épargne et investissement nationaux une estimation du degré d'intégration financière internationale.
} 


\section{III.2. Les FTN : principal moteur de la mondialisation}

Les FTN permettent de pousser plus loin la mondialisation. C'est ainsi qu'on a constaté une croissance rapide des flux d'IDE depuis les années 70 et qui a vraisemblablement tiré la croissance des échanges mondiaux.

Ces IDE renforcent les spécialisations des pays et permettent une exploitation plus approfondie des avantages comparatifs.

La fragmentation des processus de production conduite par les FTN permet de renforcer encore la DIT, et donc, à priori les gains mutuels à l'échange mis en avant par les théories du commerce international.

Donc, l'accélération de la mondialisation est poussée par le phénomène de multinationalisation.

\section{III.3. Les enjeux au niveau mondial}

\section{III.3.1. IDE et commerce international : complémentarité ou substituabilité ?}

L'essor du commerce international est concomitant d'une expansion du nombre des FTN. Longtemps, celles-ci sont penchées à la recherche d'un accès aux matières premières, de faibles coûts de main d'œuvre, puis d'une proximité avec la demande locale. Désormais, de plus en plus d'entreprises organisent leurs productions à l'échelle mondiale et génèrent de ce fait une part grandissante de commerce via les échanges entre leurs filiales. Empiriquement, la relation de complémentarité domine très souvent comme l'ont montré Lipsey et Weiss (1984). Pour Fontagné et Pajot (1999), pour un dollar investi à l'étranger par une industrie, on enregistre 58 cents d'exportations supplémentaire pour cette industrie.

\section{III.3.2. La croissance des flux d'IDE}

La tendance globale sur les trois dernières décennies montre une augmentation spectaculaire des investissements mondiaux. Le commerce international a beaucoup augmenté après les années 60 , et a connu une croissance plus élevé que le PIB mondial. Les IDE ont suivi une évolution parallèle, jusqu'au milieu des années 80, puis leur croissance a finalement dépassé largement celle, déjà forte, du commerce international.

Fig.3 : Évolution du PIB mondial, des exportations et des IDE (base 100, 1970)

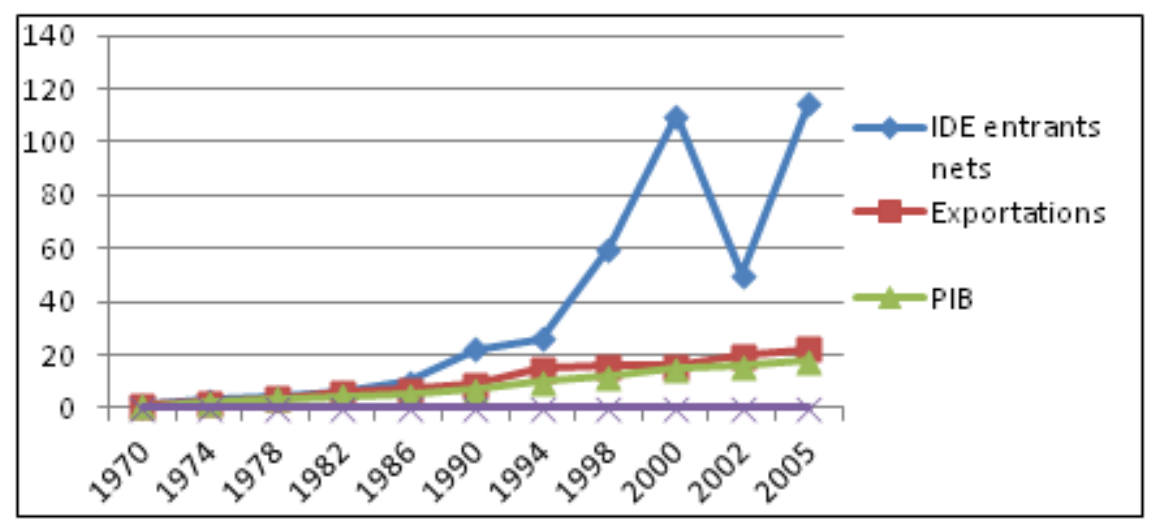

Source : Mondialisation et commerce international, Cahier Français $n^{\circ} 325$.

\section{III.3.3. Processus de multinationalisation : quel est l'ampleur du phénomène ${ }^{4}$ ?}

D'après les estimations de la CNUCED $^{5}$, il existe aujourd'hui dans le monde environ 65000 entreprises multinationales qui comptent quelques 850000 filiales étrangères dans les divers pays. En effet, le nombre d'entreprises dont l'activité s'étend au-delà des frontières s'est accru de manière exceptionnelle au cours des trois dernières décennies. Le phénomène n'est pas nouveau, mais il prend aujourd'hui une dimension sans précédente.

Cette multinationalisation qui permet de prendre les meilleurs territoires économiques tout en accédant aux différent marchés s'accompagne bien souvent d'une centralisation de certaines fonctions stratégiques, tels que la R\&D ou la finance. Il n'existe cependant pas de modèle unique, mais une grande diversité en fonction des secteurs.

Par ailleurs, la progression de l'internationalisation productive des firmes est spectaculaire. Les ventes réalisées par des filiales à l'étranger ont dépassé le volume du commerce international à partir du milieu des

\footnotetext{
${ }^{4}$ Extrait d'Alternatives Économiques numéro 1125.

${ }^{5}$ CNUCED (2004), World Investment Report, http://www.unctad.org
} 
années 80. Elle en représente aujourd'hui presque le double : 13500 milliards de dollars contre 6900 milliards. Les transnationales produisent $10 \%$ de la production mondial et contrôle les $2 / 3$ du commerce mondial. Le stock des IDE remonte à plus de $16 \%$ du PIB mondial, un niveau jamais atteint auparavant. Enfin, sous l'effet du double mouvement de dérégulation et de privatisation, les services financiers et les entreprises de réseaux (télécoms, transports,...) sont en train de s'internationaliser rapidement.

Fig.4 : La concentration du stock d'IDE des firmes transnationales en 2004

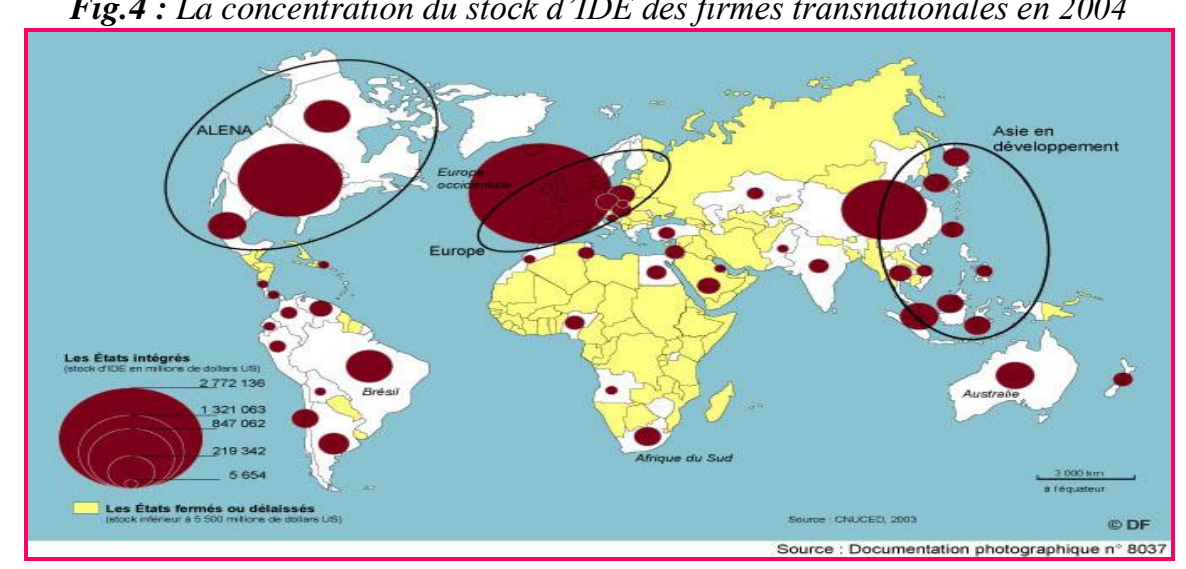

Source : Document photographique $n^{\circ} 8037$.

Toutefois, l'immense majorité des FTN conserve des relations étroites avec leurs pays d'origine. En moyenne, les FTN réalisent encore plus de la moitié de leurs ventes avec leurs nations d'origine. Mais cette proportion tend à diminuer : de $57 \%$ en 1993 à $50 \%$ aujourd'hui pour les 100 premières multinationales.

Cependant, la place des pays émergent et en développement, comme pays d'accueils des IDE, est en augmentation régulière. En 2008, les IDE entrants dans les pays développés diminuent alors que pour les PED et les économies en transition ils continuent à augmenter. Ce dernier groupe de pays voit sa part près de $43 \%$. Les pays les moins avancés voient leur part augmenter de $2 \%$ seulement. L'Asie du Sud est le grand récepteur : près de la moitié du total des IDE entrants dans les PED ${ }^{6}$.

Plus encore que le commerce international, les flux d'IDE sont le fait des pays développés : l'ensemble des flux d'IDE consiste en des flux Nord-Nord. Il s'agit de flux croisés d'investissement entre pays riches et proches (la Chine est une exception). Notons aussi le fait qu'en 2008, les IDE sortent non seulement des pays développés mais encore de l'Asie Est.

Ainsi, l'évolution des flux d'IDE traduit l'extension du poids des FTN. Ces investissements augmentent rapidement si bien que la mondialisation semble davantage portée aujourd'hui par l'accroissement des IDE que par l'ouverture commercial des pays.

Fig.5: Flux d'IDE global, 2004-2011

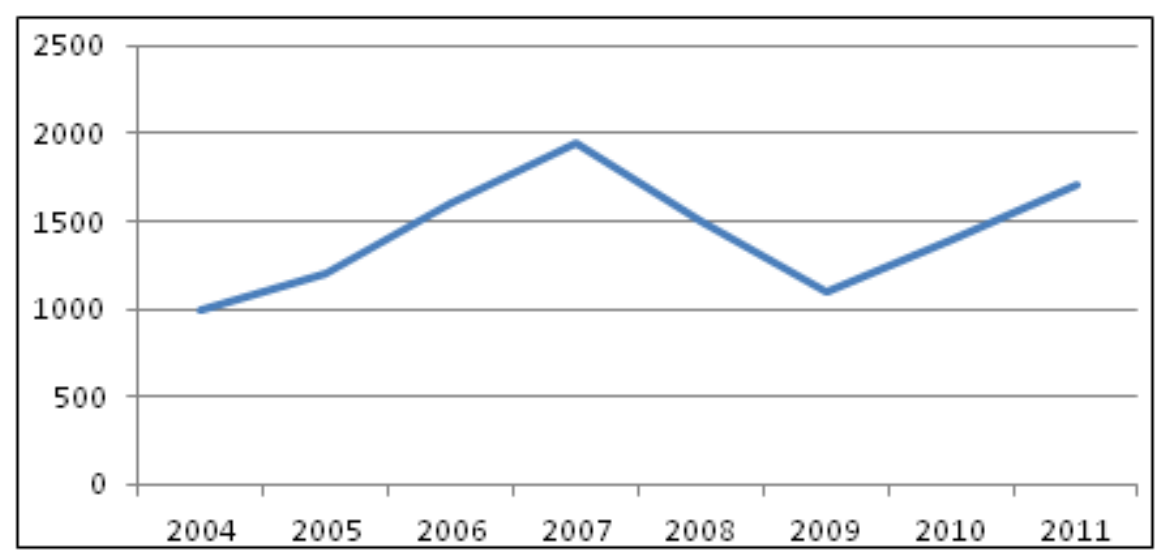

Source: UNCTAD.

${ }^{6}$ CNUCED (2009), World Investment Report 2009, transnational corporations, agricultural production and development. 


\section{Les Conséquences des délocalisations sur l'emploi \\ IV.1. Dynamique de l'impact des délocalisations ${ }^{7}$}

Le phénomène des délocalisations est le résultat d'interaction entre l'investissement direct, la soustraitance avec des firmes non affiliées et le commerce international.

En général, l'investissement direct implique des flux commerciaux notamment des importations vers les pays d'origine mais également des exportations.

La dynamique de ces interactions peut engendrer des effets positifs ou négatifs sur l'emploi de manière directe ou indirecte et selon qu'il s'agit du court ou du long terme.

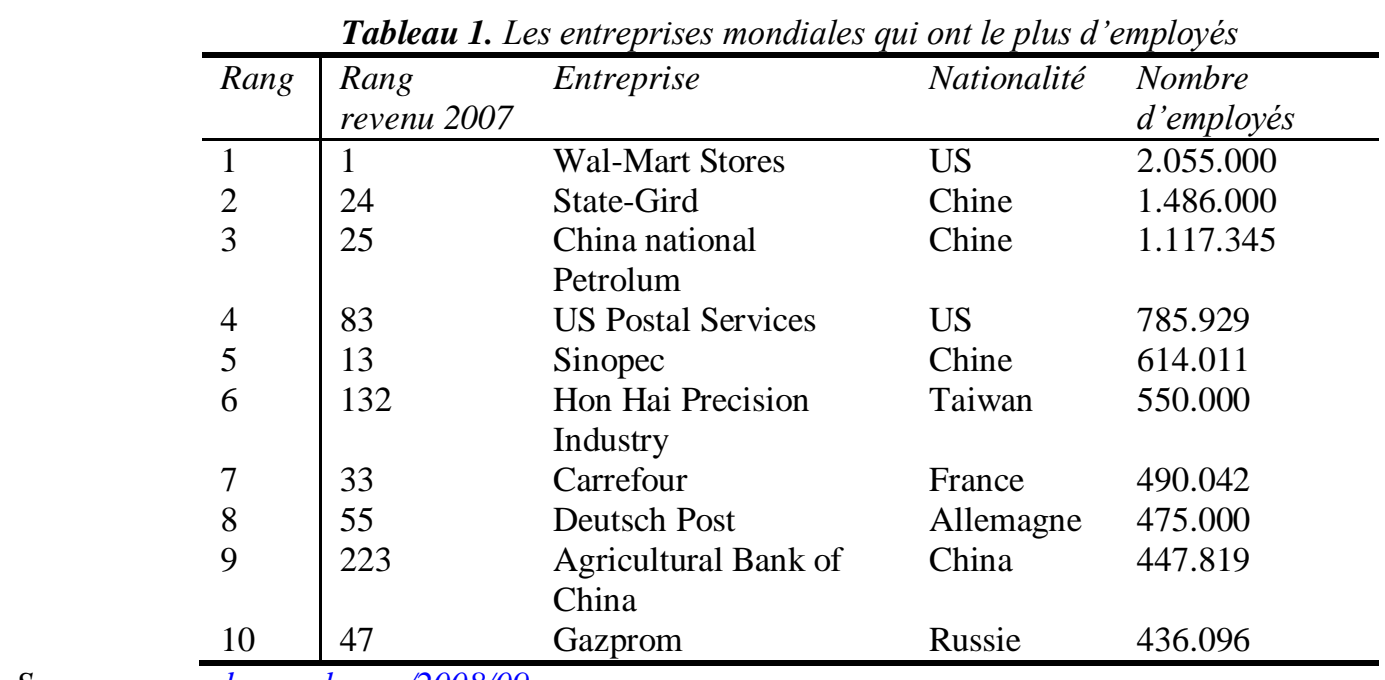

Source: www.chrogeak.com/2008/09

On remarque que la nomenclature entre le classement par revenu et le nombre d'employés est différente. On note aussi le grand nombre d'anciennes entreprises d'Etat.

Par ailleurs, les États-Unis ont le plus grand nombre d'entreprises avec un nombre de 7 entreprises dans les vingt premières ; mais la Chine est très bon deuxième avec 6 entreprises.

\subsection{L'impact des délocalisations sur l'emploi dans les pays développés}

Phénomène en progression, corollaire et générateur de la mondialisation de l'économie, les délocalisations se trouvent au cœur des problématiques de l'emploi. Délocaliser, c'est séparer les lieux (ou pays) de fabrication ou de transformation des marchandises de leurs lieux (ou pays) de consommation.

La production délocalisée s'effectuant en dehors du territoire national, elle offre ses emplois aux résidents des pays d'accueil. C'est pourquoi les délocalisations sont avancées pour expliquer, en partie, la montée du chômage dans certains pays. Pour l'entrepreneur, la délocalisation répond à une contrainte de gestion: produire là où c'est le moins cher et vendre là où il $\mathrm{y} a$ du pouvoir d'achat.

Le transfert à l'étranger de l'ensemble des activités d'une production donnée ou bien de certaines d'entre elles, constitue un thème majeur des débats publics et académiques au sein des pays. Les questionnements entourant ses impacts sur l'économie domestique rejoignent plus largement celles concernant l'impact de la mondialisation sur l'emploi et les revenus.

Afin de fournir un cadre théorique cohérent pour étudier la plus fine division du travail à l'œuvre à travers la fragmentation internationale du processus productif et son impact sur les prix, l'emploi et les revenus , Gene M. Grossman et Esteban Rossi-Hansberg (2008) ont modélisé une économie à deux secteurs et incorporant deux types de travailleurs aux niveaux de qualifications différents. Ils ont montré que la délocalisation est propre à générer un effet productivité qui bénéficie au facteur dont les tâches sont transférées à l'étranger.

\section{IV.2.1. Inquiétudes des pays développés}

\footnotetext{
${ }^{7}$ T. Hatzichoronoglou a fait beaucoup d'études sur les conséquences de l'emploi.
} 
L'effet des délocalisations sur l'emploi est devenu un thème majeur et récurrent dans le débat public. Fortement médiatisé aujourd'hui, le phénomène n'est pourtant pas nouveau. Mais il semble s'accélérer sous l'effet de la progression de la mondialisation.

On s'interrogeait déjà sur le danger que représentait la concurrence des pays émergents pour l'industrie des pays industrialisés.

Fig.6 : Pertes d'emplois dues aux délocalisations par secteur en 2005 dans l'UE (\%par rapport aux pertes totales)

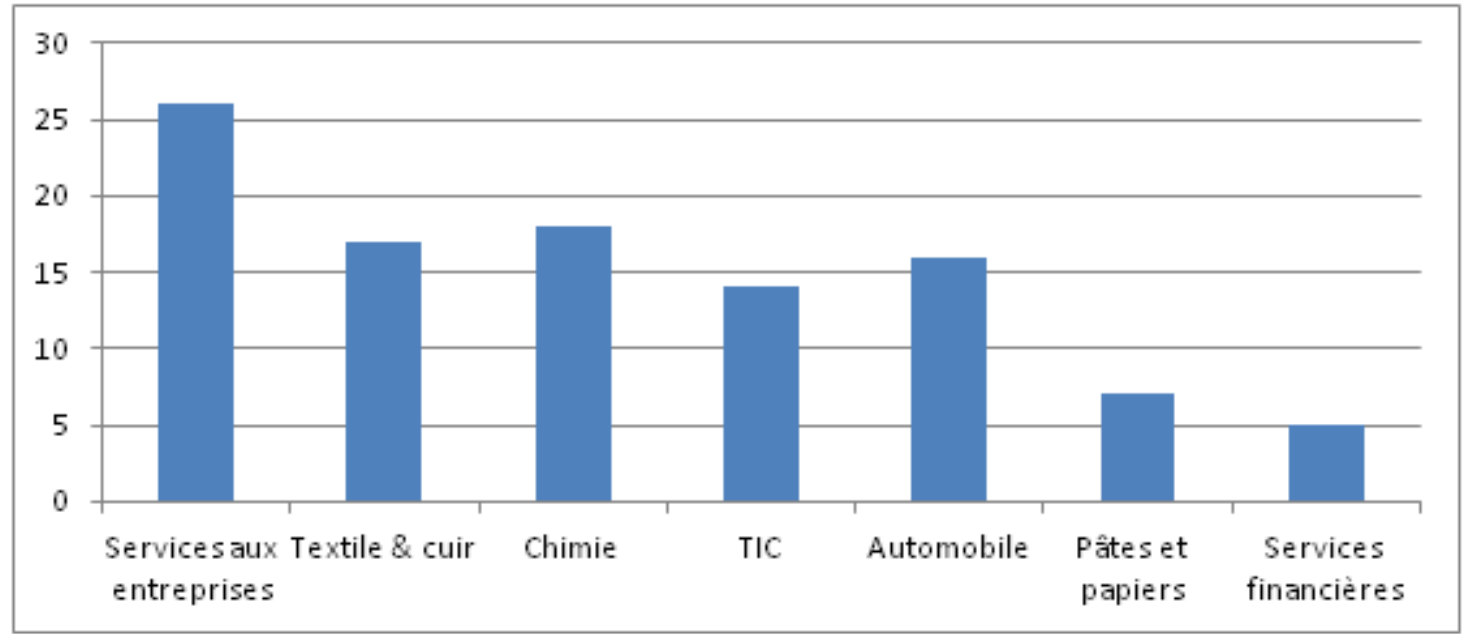

Source : Commission européenne, ERM, 2005.

Ce phénomène, suscite des inquiétudes parce que désormais, il ne concerne plus uniquement les secteurs traditionnels de l'industrie manufacturière et les emplois peu qualifiés, mais également les secteurs technologiquement plus avancés et les services, notamment les services destinés aux entreprises. Plus récemment, les emplois plus qualifiés ont également été touchés par les délocalisations. À ces raisons s'ajoute l'émergence de deux grandes économies d'Asie, la Chine et l'Inde qui, ayant rattrapé en partie leur retard technologique, disposent d'une main d'œuvre abondante et de plus en plus qualifiée.

Si les secteurs relativement protégés comme les services informatiques, ne sont plus à l'abri de la concurrence internationale, cela accroît les inquiétudes des personnes qualifiées qui peuvent perdre leurs emplois, mais surtout des personnes non qualifiées dont les emplois deviennent plus vulnérables. En d'autres termes, la mobilité des capitaux aboutit à la création des emplois peu qualifiés dans les pays à bas salaires, ce qui se traduit par l'inélasticité de la demande du travail dans les pays développés. On croit qu'avec le rapatriement des dividendes, les pays développés récoltent une perte nette d'emplois en termes d'avantage comparatifs.

\section{IV.2.2. La mondialisation transforme-t-elle vraiment les emplois vers les pays à bas salaires? \\ IV.2.2.1. Analyse préliminaire}

Cet impact peut être saisi à partir de deux filières : la délocalisation des entreprises et la division internationale du travail (DIT) entre la maison-mère et les filiales.

$>$ filière 1 : délocalisation des entreprises et contenu en emploi

En ce qui concerne l'impact de l'IDE sur le pays d'origine de l'investissement, celui-ci a souvent être saisie indirectement à partir des échanges engendrés par le transfert d'activité à l'étranger.

D'un côté, il existe les effets de déplacement de l'emploi compte tenu du fait que les produits importés auraient pu être des produits crées localement. D'un autre côté, il existe des effets de création ou de destruction lié à la stimulation ou à la diminution des exportations suite à l'implantation. Il s'agit de calculer le contenu en emploi des flux commerciaux net engendrés par les implantations à l'étranger des FTN.

$>$ filière2 : la DIT

Une façon de traiter la relation IDE et emploi est d'envisager ce lien comme une résultante de la DIT mise en place par la maison-mère à l'intérieur de son réseau de filiales.

La maison-mère peut se spécialiser dans quelques segments productifs et abandonner certaines tâches au profit de ces filiales à l'étranger. Donc, on s'intéresse à la question de la répartition du travail entre les différentes unités de productions existantes dans la FTN.

En effet, il existe deux types d'analyse qui traitent de l'impact de la DIT dans la FTN : 
- L'évaluation en relation de substitution ou de complémentarité entre les travailleurs nationaux et les travailleurs étrangers ;

- La recherche de l'impact de l'IDE sur la qualification de l'emploi dans la maison-mère.

\section{IV.2.2.2. Théoriquement, les délocalisations ne provoquent pas des diminutions d'emplois}

Le jeu normal de la spécialisation des économies fait que les délocalisations ne diminuent pas le nombre d'emplois.

En effet, le Sud capte l'essentiel des productions traditionnelles (textile, cuir, chaussures, électronique grand public, etc.) car il a une structure économique adaptée à ce type de production, qui demande beaucoup de main d'œuvre non qualifiée et peu de technologie innovante. Le Nord se concentre sur les activités élaborées qui réclament technologie et savoir-faire.

Ainsi, les facteurs de productions libérés dans les secteurs importateurs sont alors récupérés par les secteurs exportateurs en pleine croissance, garantissant ainsi le plein-emploi.

\section{IV.2.2.3. Statistiquement, aussi non}

$>$ Un phénomène mal mesuré :

Des rapports récents sont venus alimenter le débat sur les délocalisations. Ils concluent à une faible ampleur de ce phénomène et le replacent dans une perspective plus large d'ouverture économique. Tous ces rapports soulignent néanmoins une des faiblesses du diagnostic établi, qui tient à l'absence de mesure précise du phénomène. Dans la quasi-totalité des études, l'ampleur des délocalisations n'est en effet appréciée que de manière indirecte, en regardant par exemple le poids des pays émergents dans les investissements ou les importations des pays développés. Il n'existait pas jusqu'à présent de chiffrage précis en termes d'emplois ou d'établissements touchés.

$>$ Les vraies causes de la réduction d'effectifs employés

Selon Mathieu et Sterdyniak (1992), les nouveaux pays industrialisés (NPI) seront responsable de moins de $1 \%$ de chômage de la population active au Nord, ce qui est un taux négligeable. Aucune étude ne démontre de façon convaincante des pertes nettes d'emplois importantes associées au commerce international et aux délocalisations.

En France, seuls 4\% des investissements à l'étranger correspondraient effectivement à des délocalisations selon la $\mathrm{DRCE}^{8}$. Tandis que l'INSEE ${ }^{9}$ montre que les délocalisations (y compris la sous-traitance) détruiraient 13500 emplois par an, chiffre négligeable au regard des 10000 emplois détruit chaque jour ouvrable par l'économie française.

Selon l'Observateur EMCC de Dublin, les délocalisations sont responsables de moins de 5\% des réductions totales des postes en Europe, loin derrière les faillites, fermeture ou restrictions.

Les études réalisées jusqu'à présent montrent que les pertes d'emploi dues aux délocalisations ne représentent qu'un très faible pourcentage dans le total des emplois détruits.

Dans ces conditions, il pourrait paraître paradoxal qu'il y ait autant de débats et de crainte dans de nombreux pays à cause des délocalisations.

Fig.7 : Les causes de la réduction d'emplois en Europe

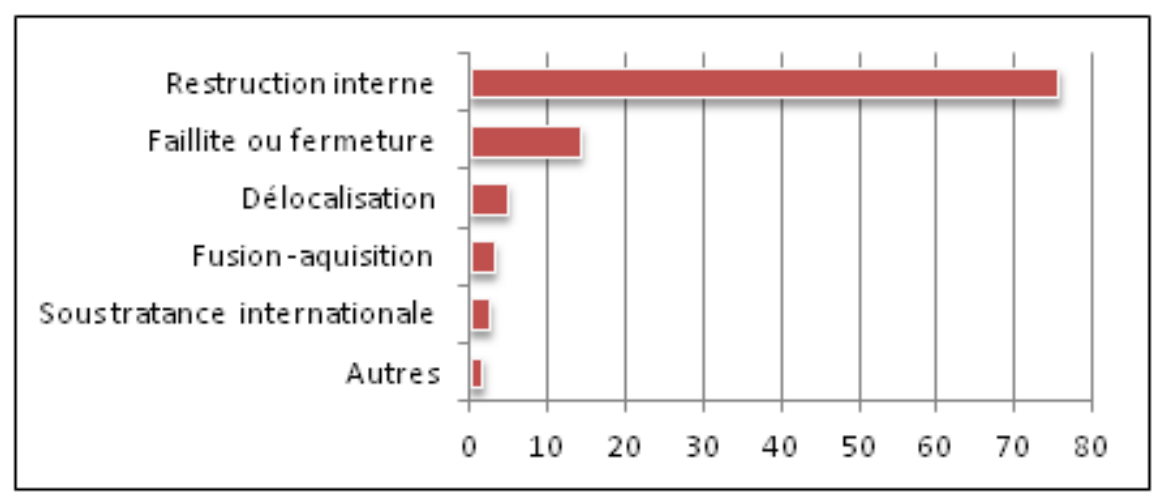

Source : Observateur EMMC/Dublin, 2007.

\footnotetext{
${ }^{8}$ Direction Régionale du Commerce Extérieur en France, site web : www.dree.org/drce

${ }^{9}$ Institut National de la Statistique et des Etudes Economiques, site web : www.insee.fr
} 


\section{IV.2.3. Les bénéfices de la délocalisation}

\section{IV.2.3.1. Bénéfices des délocalisations dans un modèle à un seul bien ${ }^{10}$}

Ce modèle comporte un seul bien et deux facteurs de production, le travail et le capital. Soit $\mathrm{L}^{0}$ la dotation du travail et $\mathrm{W}^{0}$ le salaire de la main d'œuvre.

Fig. 8 : Bénéfices des délocalisations dans un modèle à un seul bien

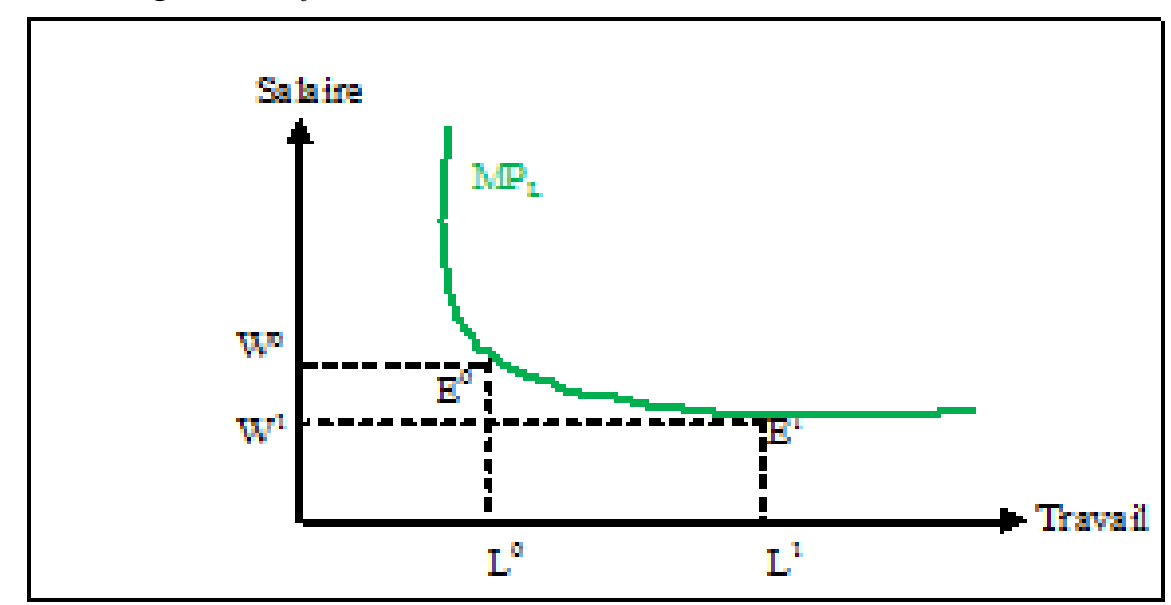

Source: Hatzichoronoglou T., L'impact des délocalisations sur l'emploi : problèmes de mesure et implications politiques, OCDE, Paris, 2006, p22

On suppose que ces facteurs ont des rendements décroissants et que la dotation en capital est fixe dans l'économie. L'existence d'un seul bien exclut au départ le besoin du commerce.

Dans le schéma, la courbe $\mathrm{MP}_{\mathrm{L}}$ représente le produit marginal du travail. La masse salariale est la zone formée par le rectangle $\mathrm{OW}^{0} \mathrm{E}^{0} \mathrm{~L}^{0}$.

Supposons qu'une innovation permette à l'économie d'acheter électroniquement des services de main d'œuvre étrangère au taux fixe de salaire $\mathrm{W}^{1}$. L'économie continue d'employer la même dotation de main d'œuvre nationale mais en la rémunérant désormais avec un salaire inférieur. Dans ce cas, l'économie achète le travail $\mathrm{L}^{0} \mathrm{~L}^{1}$ à l'étranger en le payant la somme correspondant au rectangle $\mathrm{L}^{0} \mathrm{~L}^{1} \mathrm{E}^{1} \mathrm{R}$.

La main d'œuvre nationale reçoit la somme correspondante à $\mathrm{OL}^{0} \mathrm{RW}^{1}$ et le capital celle située en dessous de la courbe $\mathrm{MP}_{\mathrm{L}}$ et au-dessus de la ligne horizontale $\mathrm{W}^{1} \mathrm{E}^{1}$.

Il en résultera les effets économiques suivants :

- Le revenu total du pays augmentera du triangle $\mathrm{E}^{0} \mathrm{RE}^{1}$, ce qui représente le gain de la délocalisation d'activités ;

- Le revenu du travail, le facteur «concurrençant les importations» sera amputé de la zone $\mathrm{W}^{0} \mathrm{E}^{0} \mathrm{RW}^{1}$ pour être redistribué au capital ;

- Les détenteurs des capitaux vont acquérir donc un gain qui correspond à $\mathrm{W}^{0} \mathrm{E}^{0} \mathrm{RW}^{1}$.

Ce modèle montre que la délocalisation peut s'avérer bénéfique pour l'économie dans son ensemble. Mais en l'absence de mécanismes permettant de transférer aux salariés certains bénéfices sociaux reçus par le capital, ce sont les entreprises et les détenteurs de capitaux qui reçoivent plus que $100 \%$ des bénéfices des délocalisations alors que les travailleurs n'en subissent que des pertes.

\section{IV.2.3.2. Réalités des bénéfices}

$>$ Une gamme de profits économiques

McKinsey Global Institute (MGI) ${ }^{11}$ a quantifié dans une étude les bénéfices économiques que les FMN ont pu tirer de la délocalisation des services à l'étranger. Ces bénéfices sont mesurés par 1 dollar US délocalisé à l'étranger dans le secteur des services.

Les États-Unis rapatrient :

- 0.58 USD dus aux réductions des coûts de main d'œuvre ;

\footnotetext{
${ }^{10}$ Hatzichoroglou T. (2006).

${ }^{11}$ La mission de MGI est d'aider les dirigeants dans les secteurs commerciaux, publics et sociaux à développer une meilleure compréhension de l'évolution de l'économie.
} 
- $\quad 0.05$ USD grâce aux exportations supplémentaires ;

- 0.05 USD correspondant aux profits rapatriés ;

- 0.47 USD correspondant aux gains dus au réemploi des personnes touchés par les délocalisations.

Fig.9 : Bénéfices provenant de 1 USD délocalisé dans le secteur des services en 2008

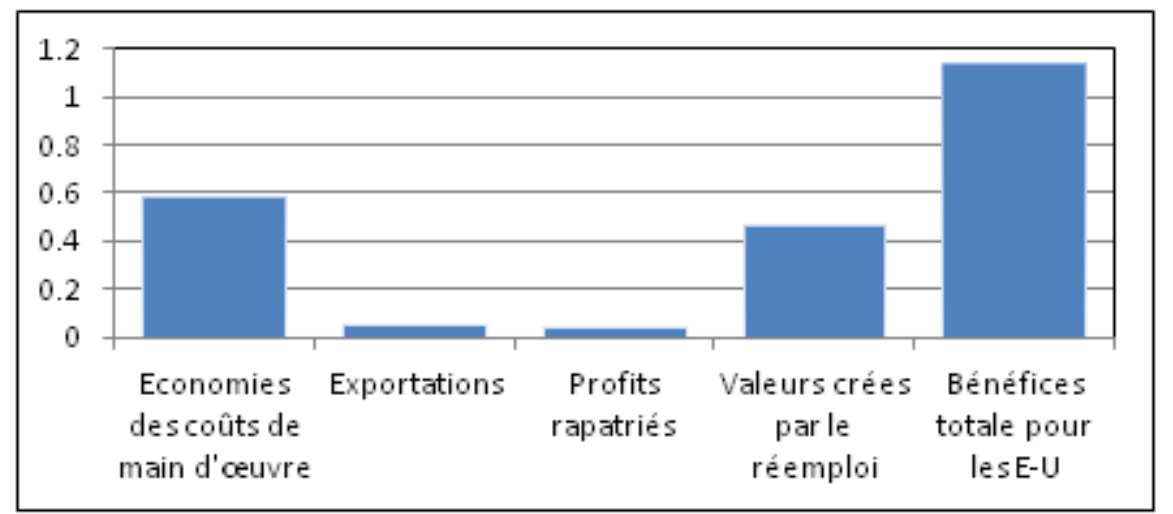

Source: McKinsey Global Institute studies, 2008.

Les mêmes évaluations pour la France et l'Allemagne donnent fait que pour 1 EUR délocalisé, la France rapatrie 0.86 EUR et l'Allemagne 0.74 EUR.

$>$ Èlargissement des marchés

Un autre aspect de la problématique des délocalisations est l'émergence de nouveaux marchés. Les pays émergents ne sont pas seulement des concurrents dont les coûts de production seraient plus faibles que ceux des pays développés. Ils sont aussi clients des pays industriels et leur demande devrait s'accroître à mesure que les revenus qu'ils tirent du commerce international augmentent et que leur développement économique s'accélère.

L'implantation des groupes européens et américains dans les pays émergents relèverait en partie d'une logique de conquête de marché, et pas seulement de diminution des coûts de production. La production y serait avant tout destinée à servir les marchés locaux, et non à être réimportée vers les pays développés. En ce sens, elle ne se substituerait pas, pour l'essentiel, à la production initialement effectuée dans ces pays développés : les délocalisations ne seraient en fait qu'une petite partie des implantations dans les pays émergents. Ceci nuance quelque peu les craintes concernant les délocalisations qui porteraient sur l'ensemble du système productif des pays industrialisés. L'émergence des pays à bas salaires représenterait aussi de nouveaux débouchés pour les entreprises des pays développés.

D Un nombre relativement limité d'emplois touchés :

Les emplois délocalisés ne sont pas exactement synonymes de licenciements. Les salariés travaillant dans un établissement dont la production est délocalisée ne sont pas systématiquement licenciés : ils peuvent être repris dans d'autres établissements du groupe ou bien dans d'autres entreprises de la même zone d'emploi. On peut avoir une première idée de la différence entre le nombre d'emplois délocalisés et le nombre d'emplois réellement détruits suite à une délocalisation en observant l'évolution des effectifs employés par les groupes sur les zones d'emploi où les délocalisations ont lieu. Le nombre d'emplois détruits annuellement suite aux délocalisations serait alors plus faible.

Part faibles des pays à bas salaires (PBS) dans le total des destructions d'emploi

Les destructions d'emplois les moins qualifiés concernent surtout les délocalisations vers les pays à bas salaires où les multinationales trouvent des coûts faibles de la main d'œuvre ce qui leur permet de rester compétitives (34\% des emplois détruits sont des emplois ouvriers non qualifiés). Au contraire les délocalisations vers les pays développés qui sont établis par les multinationales dans l'intérêt de trouver de la clientèle à fort pouvoir d'achat tout en contournant les mesures de protectionnisme, détruisent relativement plus d'emplois qualifiés (35\% des emplois détruits sont des emplois de professions intermédiaires et $12 \%$ sont des emplois de cadres).

Parmi les emplois délocalisés, un peu moins de la moitié le sont à destination des pays à bas salaires. Ces pays représentent environ 6400 emplois délocalisés par an, soit $0,17 \%$ de l'emploi industriel hors énergie, ou encore $6 \%$ des fortes diminutions d'effectifs.

Notons aussi le fait qu'environ 1 fermeture d'établissement industriel sur 280 correspondrait à une délocalisation vers un pays à bas salaire, soit un peu moins d'un emploi détruit sur vingt. Si on restreint le 
champ aux seuls groupes pérennes, environ 1 fermeture d'établissement sur 150 est \&une présomption de délocalisation, représentant un peu plus d'un emploi détruit sur dix.

Les influences économiques, sociales et culturelles que les multinationales exercent sur le monde pauvre. Il constate que celles-ci utilisent leur argent, leur taille et leur pouvoir pour peser sur les négociations internationales et qu'elles profitent du courant des privatisations pour orienter les politiques des gouvernements. Par ailleurs, le volume des investissements des pays avancés dans les pays en retard ne représente que $0.5 \%$ du PIB des pays industrialisés.

$>$ L'externalisation des fonctions périphériques

De plus, le recentrage des établissements industriels sur leurs activités premières a aussi pour conséquence l'externalisation des fonctions périphériques telles que les services généraux, la comptabilité, l'entretien, la maintenance ou la logistique, le recouvrement des créances, vers des prestataires de services. Aussi, à côté d'une croissance en emplois qui lui est propre, le secteur tertiaire bénéficie largement de ces deux effets : externalisation et recours à l'intérim du secteur industriel.

Ces investissements récupèrent du profit pour le pays d'origine du capital, que l'entrepreneur peut rapatrier à tout moment, ou les placer pour agrandir son patrimoine. Donc, les destructions d'emploi sont à nuancer puisque indirectement les délocalisations peuvent entraîner des créations d'emploi.

$>$ L'accroissement des revenus des consommateurs

Les emplois éliminés immédiatement peuvent être compensé par des emplois recrées sur le territoire national (Europe) par les profits issus de la délocalisation. Notons que cela dépend aussi des emplois crées grâce au supplément du pouvoir d'achat qu'obtienne les consommateurs qui achètent désormais des produits moins chers des NPI. D'où on dégage une interférence entre les niveaux microéconomiques et macroéconomiques. Cependant, au total, il semble plus juste de raisonner à un niveau macroéconomique.

Fig.10 : Gains potentiels de revenu par habitant dus aux importations des biens délocalisés (USD, moyenne 2003-2005)

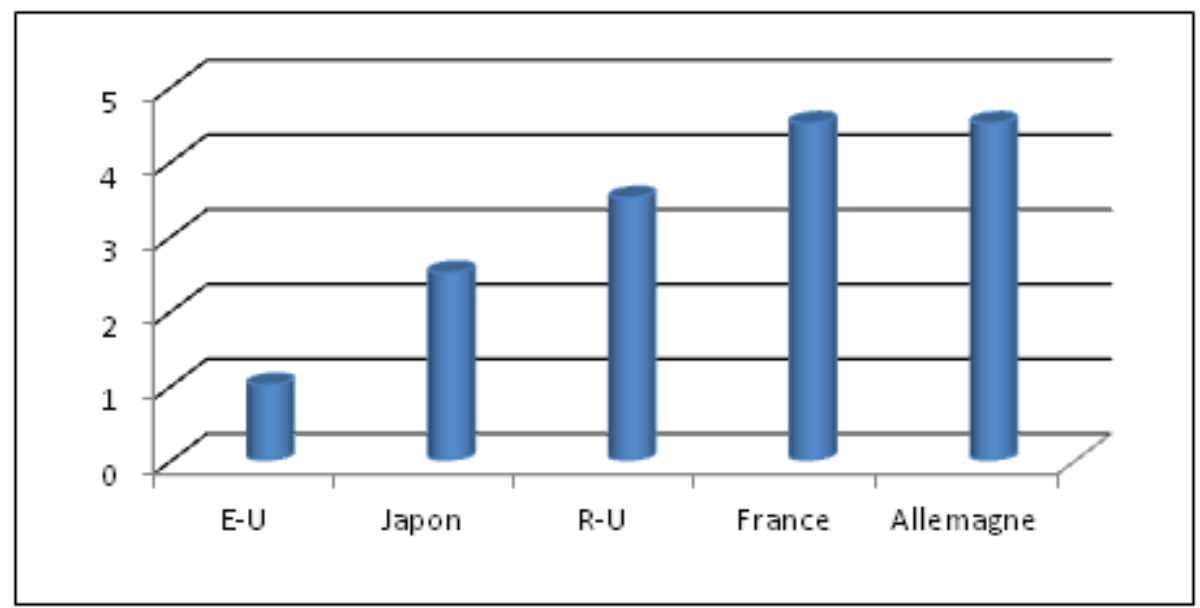

Source : CEPII, 2006.

Ainsi, acheter plus de biens en Europe va conduire à une augmentation de la demande effective ${ }^{12}$. D'où la création de plus d'emploi. Il faut aussi évaluer la diminution des exportations d'Europe vers des pays tiers (le Canada concurrence l'Europe en Amérique Latine).

D'ailleurs, pour que l'ouverture soit favorable à tous, l'Etat doit verser une allocation aux travailleurs non qualifiés et subventionne l'emploi dans les secteurs concurrencés, c'est-à-dire corriger les conséquences sociales.

De toute façon, il s'agit là d'un raisonnement dynamique.

Les délocalisations sont au cœur du débat sur la mondialisation, pour autant, pour beaucoup d'économistes, ceux-ci ne constituent pas un problème important pour les pays développés. Alors, Il serait excessif de conclure que les différences de coûts de la main d'œuvre devraient à terme à la délocalisation de l'ensemble de l'industrie des pays développés vers les pays à bas salaires.

Ces études ont montré que prétendre que l'investissement dans les pays émergents affecte le travail peu qualifié dans les pays développés relève de la pure démagogie. Au contraire, les délocalisations peuvent engendr

${ }^{12}$ La demande effective est une demande anticipée par les entrepreneurs. 
er une évolution de la compétitivité et de la productivité des entreprises, un accroissement des exportations, une maitrise de l'inflation et une meilleure rémunération des capitaux.

Le dernier rapport de la $\mathrm{CNUCED}^{13}$ sur l'investissement dans le monde révèle que la Chine est largement le premier pays d'accueil envisagé des futurs centres délocalisés de R\&D entre 2006 et 2009. Mais le développement du Sud ouvre également des opportunités extraordinaires aux entreprises du Nord. Tout dépend alors des performances de chacun sur ces marchés émergents.

Donc, la vraie question pour les pays développés est de savoir s'ils peuvent pleinement profiter de l'essor de ces pays et ne pas seulement subir leur concurrence.

\section{IV.3. Impacts des délocalisations sur l'emploi des pays hôtes}

IV.3.1. Deux impacts majeurs

Il s'agit précisément de savoir qu'elle est l'impact de l'implantation de la FTN sur le niveau d'activité investi dans un secteur dans le pays hôte.

Markusen et Venoble (1999) soulignent deux impacts majeurs de la délocalisation sur l'emploi dans les pays qui accueillent les FTN: l'effet pro-concurrentiel et l'effet amont avec les producteurs de biens intermédiaires.

\section{IV.3.1.1. L'effet pro-concurrentiel}

Théoriquement, l'effet pro-concurrentiel des délocalisations a suscité beaucoup de débat de la part des économistes. L'implantation de firmes multinationales renforce la pression concurrentielle. C'est d'ailleurs un argument fort en faveur de l'implantation : les firmes étrangères investissent dans le pays hôte afin de capter des parts de marché aux firmes locales. Les conséquences en termes d'emploi sont ambigües: d'un côté, les firmes locales peuvent réduire leur activité voire disparaître; de l'autre côté, l'entrée de firmes étrangères peut accroître l'emploi (peut être peu si la technologie est moins intensive en mains d'œuvre).

\section{IV.3.1.2. Les liens amont avec les producteurs de biens intermédiaires}

L'impact des entreprises étrangères ne peut se limiter à la modification de la structure du marché du bien final ; en plus, elles vont manifester une demande croissante des biens intermédiaires du pays hôte.

Fig.11 : Effet d'entrainement de l'IDE

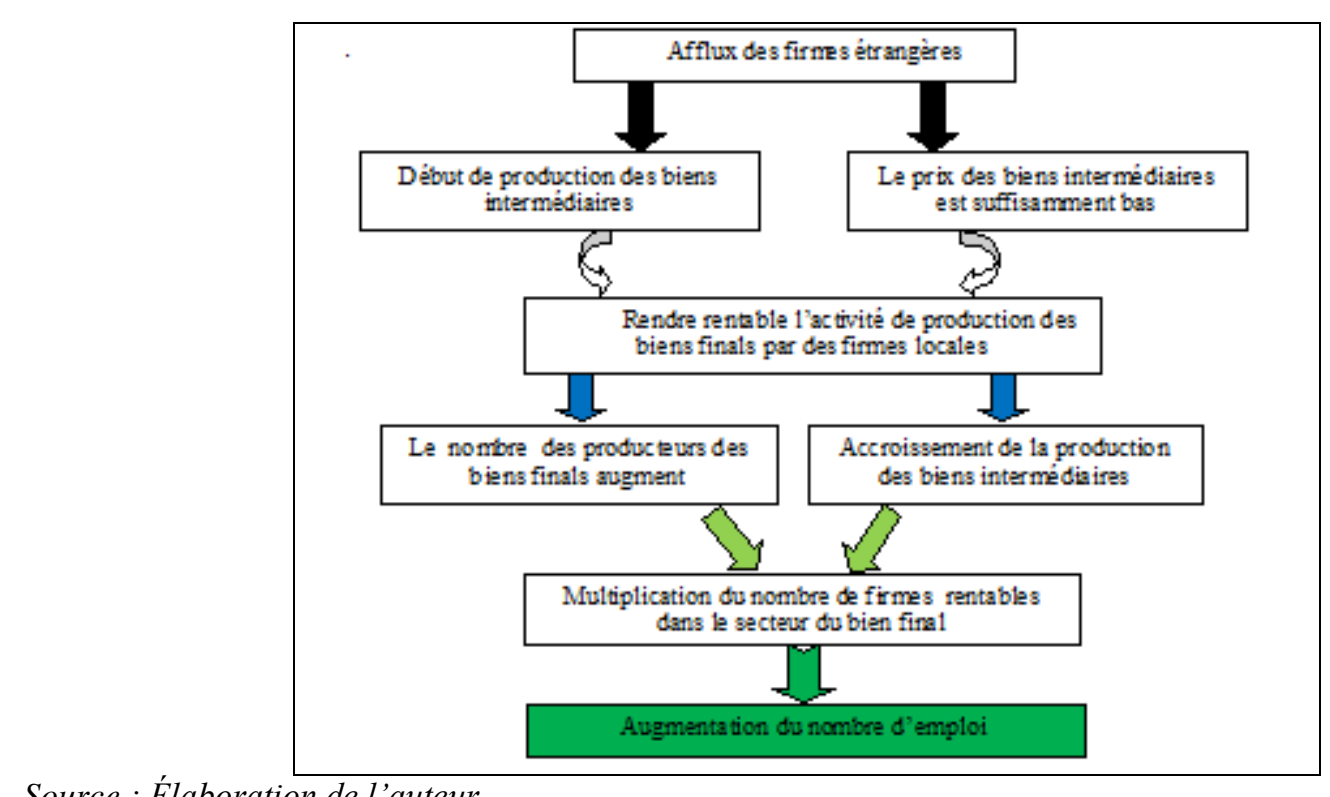

Source : Élaboration de l'auteur.

À noter que si, en plus, le secteur producteur de biens intermédiaires présente des économies d'échelle; la hausse de la production dans ce secteur va provoquer une baisse du prix de vente qui va profiter aux producteurs de biens finals. Ainsi, ce mécanisme est cumulatif.

L'impact total de l'implantation des FTN va dépendre de l'ampleur de chacun de ces deux effets sur le secteur et plus particulièrement du degré d'utilisation des biens intermédiaires locaux par les firmes du pays hôte. Si les FTN remplaçant les firmes locales utilisent moins d'intrants (inputs) locaux, l'effet de la contraction de l'activité sera renforcé. En revanche, si les FTN utilisent davantage de biens intermédiaires que les firmes

${ }^{13}$ CNUCED (2009). 
locales, l'effet lien amont peut compenser l'effet intensification de la concurrence. À la limite, l'IDE pourrait même être un catalyseur de développement.

Beaucoup d'études ont montré que l'impact de L'IDE a été initialement (à court terme) négatif sur l'entrée des firmes locales puisque les effets de concurrence dominent les effets d'entrainement. Cependant, cet effet négatif est ensuite dominé (à long terme) par les effets positifs.

\section{IV.3.2. Les IDE amorcent l'activité et l'emploi}

Il est généralement avancé que les IDE ont des effets positifs substantiels pour les pays récepteurs à travers la diffusion d'externalités horizontales et verticales.

En effet, les IDE provoquent des effets positifs sur quelques variables moteurs de la croissance à savoir : l'investissement domestique, le commerce, le transfert de la technologie, le capital humain. La théorie de croissance endogène ${ }^{14}$ est un cadre approprié pour l'étude de l'effet de l'IDE sur la croissance économique.

$>$ Les pays émergents à la conquête des marchés mondiaux

De grandes firmes de services informatiques se sont développées et commencent à prendre pied sur les marchés des pays développés.

Nous discutons ci-dessus l'exemple de l'industrie du logiciel qui a connu depuis 15 ans une croissance spectaculaire en Inde comme en Chine. D'un côté, l'Inde est aujourd'hui l'un des leaders mondiaux des exportations de services informatiques. Selon le Rapport sur le Commerce Mondial de 2005, elle se classe au deuxième rang mondial (derrière l'Irlande) avec $15 \%$ du total des exportations dans le secteur. D'un autre côté, l'industrie du logiciel est encore dans une phase émergente en Chine (OCDE, 2007).

Tableau 2. Chiffres d'affaire de quelques firmes de fabrication des logiciels(en milliers d'euros)

\begin{tabular}{llc}
\hline Pays & Nom & CA en 2007 \\
Inde & Wipro & 2.596 .410 \\
Inde & Infosys Technologies & 2.397 .359 \\
Inde & Tata Consultancy Services & 2.471 .289 \\
Chine & Xiaman Xinde & 533.037 \\
Chine & Tsinghuan Unisplondour & 340.391 \\
É-U & Microsoft Corporation & 37.942 .748 \\
France & Atos Origin & 5.396 .900 \\
R-U & LogicaCmg PLG & 3.955 .789 \\
\hline
\end{tabular}

Source : Bases de données ZEPHYR et ORBIS, 2008.

Par ailleurs, l'afflux d'investissements étrangers a créé en Chine des industries très dynamiques qui ont accéléré la croissance économique et qui lui ont assuré des gains de parts de marchés spectaculaires dans les échanges mondiaux. La participation croissante de la Chine au commerce international a été directement liée à son insertion dans une division internationale des processus de production en Asie. Les stratégies de délocalisation des firmes étrangères ont développé sur le continent des entreprises exportatrices qui, initialement cantonnées à un rôle d'atelier d'assemblage, semblent gagner progressivement une maîtrise plus complète des processus de production et de commercialisation.

$>$ Des effets positifs sur les salaires

Les nouvelles données confirment que l'IDE peut avoir un effet positif important sur les salaires versés par les entreprises étrangères dans le pays d'accueil, même lorsque l'analyse se limite aux effets à court terme des fusions et acquisitions internationales. Conformément à l'opinion courante, cet avantage salarial est plus marqué dans les économies émergentes.

En outre, l'effet positif de l'IDE consiste essentiellement à offrir de meilleures possibilités d'emploi pour les nouveaux recrutés plutôt qu'un meilleur salaire aux travailleurs restant en place dans les entreprises qui changent de propriétaire. Cela peut tenir à des conditions plus concurrentielles sur le marché de l'embauche, qui permettent aux nouveaux recrutés de participer plus largement à l'avantage de productivité des FTN.

\footnotetext{
${ }^{14}$ Le premier modèle de croissance endogène a été publié par Paul Romer en 1986, dans un article intitulé Increasing Returns and Long Run Growth. Cette théorie a été développée principalement par Paul Romer, Robert E. Lucas, et Robert Barro.
} 
Fig.12: Effet moyen de la prise en contrôle étrangère sur les salaires moyens (différence en \%)

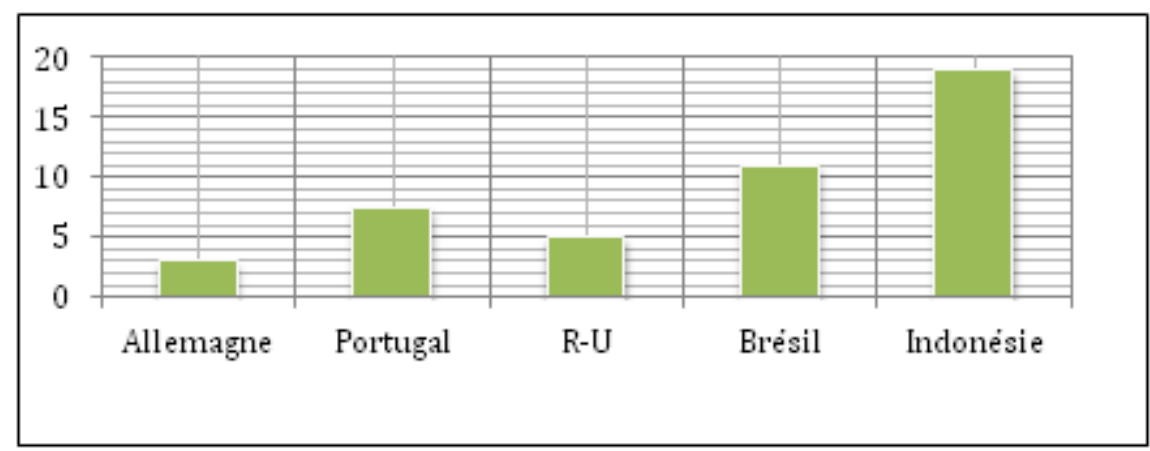

Source : Perspectives de l'emploi de l'OCDE 2008, Paris.

\section{$>$ Autres bénéfices}

Outre ses effets directs sur les salariés des multinationales, l'IDE peut avoir des effets indirects sur les conditions de travail dans les entreprises locales lorsqu'il donne lieu à des transferts de connaissances ${ }^{15}$. En effet, il peut engendrer un transfert de technologie et une diffusion des externalités positives (les spillovers). Aussi, il peut accroitre la formation du capital et la création d'emploi en fournissant aux pays hôtes des ressources spéciales telles que le savoir-faire en management, le travail qualifié, l'accès à des réseaux internationaux de production et de distribution.

\section{IV.3.3. Des recommandations de politiques économiques}

Les PED doivent adopter une approche faisant des délocalisations une composante principale de leurs schémas de développement pour profiter pleinement des IDE attirés sur son territoire.

C'est ainsi que des mesures propices à l'IDE pourraient être une composante utile d'un cadre d'action intégré en faveur du développement. En concevant certaines mesures, les pouvoirs publics auraient intérêt à tenir compte du fait qu'elles peuvent influer non seulement sur le volume des entrées d'IDE, mais aussi sur leur composition et par conséquent sur les avantages qui peuvent en découler. Dans un premier temps, on pourrait lever les dispositions réglementaires spécifiques qui font obstacle aux entrées d'IDE et peut-être aussi, dans certaines circonstances, accorder des incitations particulières aux investisseurs étrangers potentiels. Ces mesures ciblées ne remplaceraient toutefois remplacer l'effort à engager pour améliorer l'environnement des entreprises de façon générale. Par contre, abaisser les normes fondamentales du travail dans l'espoir d'offrir aux investisseurs potentiels un environnement plus compétitif a de fortes chances d'aller à l'encontre du but recherché. Non seulement il ne semble pas que cela attire l'IDE, mais cela risque de décourager les investissements des FTN responsables pour lesquelles il est important de s'assurer que des normes minimales touchant aux conditions de travail sont respectées dans l'ensemble de leurs activités. Notons que les mesures des pays d'accueil en faveur de l'IDE peuvent être utilement complétées par des initiatives multilatérales visant à renforcer les effets bénéfiques des entrées d'IDE sur le plan social en encourageant une conduite responsable parmi les FTN.

\section{Conclusion}

Les FTN sont naturellement des entreprises actives sur les marchés mondiaux. En effet, elles sont impliquées dans près de deux tiers des relations commerciales internationales. Ainsi, les réflexions sur le développement du commerce mondial ne peuvent plus négliger aujourd'hui l'influence de ces acteurs du processus d'internationalisation des économies.

Par ailleurs, les IDE ont connu une croissance plus élevée que les autres indicateurs majeurs de l'économie mondiale. Donc, les IDE ne sont pas déconnectés des FTN qui poussent plus loin la mondialisation : ils ne sont pas dissociés de la multinationalisation ; ils en sont le moyen de l'internationalisation. Notons que ces mouvements de capitaux ont d'abord lieu entre les pays riches, mais ils s'étendent de manière croissante au reste du monde en particulier aux marchés émergents.

Quant à l'impact social des délocalisations, on note que la prépondérance des FTN dans le commerce mondial n'est pas sans conséquence : en renforçant la DIT, les IDE renforcent davantage les gains mutuels à l'échange mis en avant par les théories du commerce international ; d'autre part, les choix des délocalisations posent à nouveau la question de la distribution des gains entre les catégories de travailleurs. Pour les pays

${ }^{15}$ www.oecd.org/publications/syntheses 
origines, le solde du contenu en emploi des flux d'IDE est positif. L'effet sur l'emploi est le résultat de la division du travail au sein de la FTN. Donc, l'inquiétude de ces pays quant à la concurrence des pays à bas salaires n'est pas fondée.

Quant aux pays hôtes, on remarque la confrontation d'un effet pro-compétitif et d'un effet sur la demande de biens intermédiaires. Pour de nombreux pays, l'effet total sur l'emploi est positif. Cependant, il demeure difficile de fournir une évaluation précise. De même, pour ces pays, l'attraction de l'investissement direct peut constituer un moteur de la croissance et facilite l'insertion dans l'économie mondiale. Donc, les efforts déployés pour attirer les capitaux étrangers, notamment sous forme d'IDE, sont cruciaux pour la croissance et donc pour l'emploi. Ainsi, l'IDE se présente comme une alternance nécessaire plutôt qu'un choix délibéré.

\section{BIBLIOGRAPHIE}

[1] J. L. Bivens, 2005, Truth and Consequences of Offshoring, Briefing Paper, Economic Policy Institute, $n^{\circ} 15,2005$.

[2] CNUCED, 2004, World Investment Report, http://www.unctad.org

[3] CNUCED, 2009, World Investment Report 2009, Transnational corporations, agricultural production and development, http://www.unctad.org/Templates/Page.asp?intItemID $=14655$

[4] C. Flandreau, et C. Riviere, La grande retransformation : intégration financière internationale et contrôle de capitaux", Économie Internationale, $\mathrm{n}^{0} 78,1999$.

[5] FMI (Fonds Monétaire International), Base de données http://www.imf.org/external/data.html

[6] FMI, Global Financial Stability Reports, perspectives de l'économie mondiale, Bulletin FMI.

[7] Fontagné, L., Pajot M., 1999, Investissement direct à l'étranger et échanges extérieurs : Un impact plus fort aux États-Unis qu'en France, Économie et Statistique, 6(12), 1999, 326-327.

[8] J.L. Muchielli, Relations économiques internationales, (Hachette, Paris, 2001)

[9] R.E. Lipsey, Weiss M.Y., 1984, "Foreign production and exports of individual firms", Review of Economics and Statistics, Vol.66, $\mathrm{n}^{0} 2$.

[10] V. Agrawal, et D. Farrel, Who Wins from Offshoring?, McKinsey Quarterly, no. 4, 2003.

[11] J. Arthuis, Les délocalisations et l'emploi, (Les Éditions d'Organisation, Paris, 1993)

[12] F. Sachwald, "Délocalisations : une destruction créatrice ?", Sociétal no. 44, 2004.

[13] N. Martin, et D. Farrell, 2004, "Exploding the Myths of Offshoring”, The McKinsey Quarterly Web Exclusive, July 2004, http://www.mckinseyquarterly.com/article_page.aspx?ar=1453\&L2=7\&L3=10\&srid=143\&g $=1$.

[14] R.E. Lipsey, MY. Weiss, 1984, Foreign production and exports of individual firms, Review of Economics and Statististics, 66(2), $\mathrm{n}^{\circ} 2,304-308,1984$

[15] C. Garner, Offshoring in the Service Sector: Economic Impact and Policy Issues", Federal Reserve Bank of Kansas City Economic Review, Third Quarter, http://www.kansascityfed.org/publicat/econrev/pdf/3Q04garn.pdf , 2004

[16] M.G. Gene, R.H. Esteban, Task Trade between Similar Countries, NBER Working Papers, n¹4554, National Bureau of Economic Research, Inc, 2008.

[17] T. Hatzichoronoglou, L'impact des délocalisations sur l'emploi: problèmes de mesure et implications politiques", OCDE, Document de Travail, 33(19), 2006.

[18] OCDE, "Perspectives de l'emploi de l'OCDE, Les délocalisations et l'emploi: tendances et impacts", www.oecd.org/els/employmentoutlook

[19] S. Jean, 2001, "Les effets de la mondialisation sur l'emploi dans les pays industrialisés : un survol de la littérature existante", DSTI/EAS/IND/SWP, ${ }^{\circ} 7$, Groupe de travail sur les statistiques, Direction de la Science, de la Technologie et de l'Industrie, $O C D E$.

[20] J. Arthuis, 1993, "Rapport d'information sur les délocalisations hors du territoire national des activités industrielles et de service", fait au nom de la Commission des finances, du contrôle budgétaire et des comptes économiques de la nation, Sénat.

[21] P. Aubert,et P. Sillard, Délocalisations et réductions d'effectifs dans l'industrie française", Série des documents de travail de la Direction des Études et Synthèses Économiques, INSEE, 2005.

[22] T. Gregori, "Outsourcing and Service Employment Growth in Italy", Dipartimento di Scienze Economiche Estatistiche, Università degli Studi di Trieste, 34127 Trieste.

[23] C. Mathieu, et H. Sterdyniak, L'émergence de l'Asie en développement menace-t-elle l'emploi en France? ", Observatoire et Diagnostique Économique, Paris, 55-106, 1994.

[24] J. Konings, et A. Murphy, Do Multinational Enterprises Relocate Employment to Low Wage Regions? Evidence from European Multinationals", version révisée du Document de travail CEPR no. 2972, 2005.

[25] J.R. Markusen, et A.J. Venables, FDI : A catalyst for industrial development”, European Review, n43, 1999. 